\title{
LIPSCHITZ AND DIFFERENTIABLE DEPENDENCE OF SOLUTIONS ON A PARAMETER IN A SCALARIZATION METHOD
}

\author{
ALICLA STERNA-KARWAT \\ (Received 30 July 1985; revised 2 October 1985) \\ Communicated by R. O. Vyborny
}

\begin{abstract}
This paper is concerned with a vector optimization problem set in a normed space where optimality is defined through a convex cone. The vector problem can be solved using a parametrized scalar problem. Under some convexity assumptions, it is shown that dependence of optimal solutions on the parameter is Lipschitz continuous. Hence differentiable dependence on the solutions on the parameter is derived.
\end{abstract}

1980 Mathematics subject classification (Amer. Math. Soc.): 49 A 27, 49 A 50.

\section{Introduction}

This paper is concerned with a vector optimization problem where optimality is defined through a convex cone. In order to find solutions we use a scalarization method proposed by Pascoletti and Serafini [15], which relies on a parametrized scalar problem. The question arises of how the solutions of the vector problem depend on the parameter. In [20] it is shown that for a large class of vector. optimization problems this independence is semi-continuous or even continuous. Pascoletti and Serafini have proved that in the finite dimensional case with the optimality defined through a polyhedral convex cone, we may obtain a differentiable dependence of solutions on the parameter. In this paper we set the vector optimization problem in a normed space and the optimality is taken with respect to an arbitrary convex cone. We show, under some convexity assumptions, that

(C) 1987 Australian Mathematical Society 0263-6115/87 \$A2.00+0.00 
the dependence of solutions to the vector problem on the parameter is Lipschitz continuous. Hence we derive some results concerning differentiable dependence of solutions and maximal values on the parameter (Section 5). In general, we do not impose any differentiable properties on the objective function. We assume only that the image of the objective function satisfies a convexity property. As far as maximal values are concerned we do not suppose even that the objective function is continuous (Section 3). When we turn our attention to solutions we need in general that the objective function admits locally a Lipschitz inverse (Section 4).

\section{Preliminaries}

Let $Y$ be a real linear space and let $B$ be a subset of $Y$. By span $B$ and aff $B$ we denote the smallest linear subspace and the smallest affine subspace of $Y$ containing $B$, respectively. Let $C$ be a convex cone in $Y$ (that is, $C \neq \varnothing$, $C+C \subseteq C, t C \subseteq C$ for $t>0$ ) with $i c r C \neq \varnothing$. Here icr $C$ stands for the intrinsic core of $C[8$, page 7$]$, that is,

$$
\operatorname{icr} C=\{c \in C: \forall y \in \operatorname{span} C \exists \varepsilon>0 \forall 0 \leqslant t \leqslant \varepsilon c+t y \in C\} .
$$

If $Y$ is finite dimensional icr $C \neq \varnothing$ holds for every convex cone $C$.

Following [16,17], we say that an element $e$ of a subset $B \subseteq Y$ is $C$-maximal (also called $C$-optimal or $C$-efficient) in $B$ if $\{b \in B: b-e \in C, b \neq e\}=\varnothing$, and we write $e \in e_{C}(B)$ (see, for instance, [4, 7, 10, 22]).

If $\operatorname{icr} C \neq \varnothing$ then icr $C$ is a convex cone, whenever $C$ is; and in that case $e_{C}(B) \subseteq e_{\text {icr } C}(B)$ for every subset $B \subseteq Y$. Elements of $e_{\text {icrC }}(B)$ we shall call $C$-quasi maximal.

Let $X$ be a given set and $f: X \rightarrow Y$ be a mapping. We consider the following vector maximization problem:

(P): maximize $f(x)$, subject to $x \in X$,

which means: find all $x \in X$ such that $f(x) \in e_{C}(f(X))$. Any such $x$ and $f(x)$ we shall call a $C$-maximal point and a $C$-maximal value of $f$ on $X$, respectively. Following [15] we define for (P) a family of scalar problems $P(q, q)$ where $(p, q) \in Y \times \operatorname{span} C$.

Let $\mathbf{R}$ denote the space of real numbers and let $\pi: \mathbf{R} \times X \times Y \rightarrow \mathbf{R}$ be the first projection, that is, $\pi(t, x, y)=t$ for $(t, x, y) \in \mathbf{R} \times X \times Y$. Then the problem $P(p, q)$ is defined as follows

$P(p, q)$ : maximize $\pi(t, x, y)$, over $\{(t, x, y): f(x)=p+t q+y, y \in C\}$. 
It was shown in [15] that for each $C$-quasimaximal point of $f$ on $X$ (hence any $C$-maximal one) $x_{0}$ there is a solution $(t, x, y)$ to $P(p, q)$ for some $(p, q) \in Y \times$ $\operatorname{span} C$ with $x=x_{0}$ and conversely, for every $(p, q) \in Y \times \operatorname{span} C$ and every solution $(t, x, y)$ to $P(p, q), x$ is a $C$-quasimaximal point of $f$ on $X$.

The conditions ensuring that the $x \in X$ obtained by solving $P(p, q)$ is actually a $C$-maximal point of $f$ on $X$ are given in [15, Theorem 3.7] and [20, Section 3]. We shall use some of them in a later part of this paper.

Let $Y$ be now a topological vector space. We shall assume that $f(X)-C$ is closed and convex. The assumptions guaranteeing that $f(X)-C$ is closed are discussed in [20, Section 4]. For instance, observe here that if $f(X)$ is compact and $C$ is closed then $f(X)-C$ is closed. Following [20] we denote by $m$ : $Y \times \operatorname{span} C \rightarrow \mathbf{R}$ the marginal function for the family of scalar problems $\{P(p, q):(p, q) \in Y \times \operatorname{span} C\}$, that is, $m(p, q)=\sup \{t: p+t q \in f(X)-C\}$ for $(p, q) \in Y \times \operatorname{span} C$, with the usual convention that the supremum over the empty set is $-\infty$. Let $M$ denote the effective domain of $m$; this is the set of those $(p, q)$ for which $m(p, q)$ is finite.

Let us recall that the relative interior of a subset $B$ in $Y$, which we denote ri $B$ is defined as the interior, when $B$ is regarded as a subset of aff $B$. By int $B$ we shall denote the interior taken in $Y$.

In [20, Corollary 6.1] it is shown that $m$ is continuous on $M$ at $\left(p_{0}, q_{0}\right) \in M$ whenever $\left(p_{0}+\mathbf{R} q_{0}\right) \cap \operatorname{ri}(f(X)-C) \neq \varnothing$. In this paper, we show that $m$ is actually Lipschitz continuous on some neighbourhood of $\left(p_{0}, q_{0}\right)$ in $M$, if $Y$ is a normed space. Hence we derive some differentiable dependence of solutions and maximal values to $(P)$ on the parameter $(p, q)$.

\section{Lipschitz dependence of maximal values on the parameter $(p, q)$}

In what follows $Y$ is a normed space with norm $\|\cdot\|$ and $Y \times Y$ is equipped with the norm $\left\|\left(y_{1}, y_{2}\right)\right\|=\left\|y_{1}\right\|+\left\|y_{2}\right\|$ for $y_{1}, y_{2} \in Y$. By $r B$ we denote the closed ball in $Y$ centered at 0 with radius $r>0$. If $K$ is a convex, absorbing set [8, page 13] then $\rho_{K}: Y \rightarrow \mathbf{R}$ denotes its Minkowski functional, that is,

$$
\rho_{K}(y)=\inf \{t: t>0, y \in t K\} \text { for } y \in Y \text {. }
$$

A mapping $F: G \rightarrow Y$, where $G$ is a subset of a normed space $Z$, is said to be locally Lipschitz at $z_{0} \in G$ if there exists a neighbourhood $V$ of $z_{0}$ in $Z$ and a number $L>0$ such that $\left\|F\left(z_{1}\right)-F\left(z_{2}\right)\right\| \leqslant L\left\|z_{1}-z_{2}\right\|$ whenever $z_{1}, z_{2} \in V \cap$ $G$. The mapping $F$ is said to be locally Lipschitz if it is locally Lipschitz at every $z_{0} \in G$. Here, by abusing the notion, $\|\cdot\|$ denotes both norms, in $Y$ and in $Z$ respectively. 
By | | we shall denote the usual absolute value in $\mathbf{R}$. First let us observe that we cannot expect to be able to deduce the local Lipschitz continuity of $m$ from its continuity using convexity since, in general, $m$ may be convex in no neighbourhood of $\left(p_{0}, q_{0}\right) \in M$ even if $f(X)-C$ is a closed, convex subset of $\mathbf{R}$ and $p_{0} \in \operatorname{int}(f(X)-C)$. To see this, take $\left.f(X)-C=\mathrm{l}-\infty, 1\right], p_{0}=0$ and $q_{0}=1$. Then $m(p, q)=q^{-1}(1-p)$ for $p<1$ and $q>0$. The Hessian of $m$ is not positive semidefinite at any $(p, q)$ with $p<1$ and $q>0$, hence $m$ cannot be convex on any $]-\varepsilon, \varepsilon[\times] 1-\varepsilon, 1+\varepsilon[$ with $0<\varepsilon<1$. Here and further on by $\left[z_{1}, z_{2}\right]$ and $] z_{1}, z_{2}$ [ we denote the closed and open line segment with end points $z_{1}, z_{2}$, respectively.

In Lemma 3.1 and Proposition 3.1 below $Y_{0}$ denote the maximal linear subspace parallel to aff $A$ for $A \subseteq Y$, that is, aff $A=Y_{0}+y_{0}$ for every $y_{0} \in$ aff $A$.

Lemma 3.1. Let $A$ be a convex set in a normed space $Y$ with ri $A \neq \varnothing$. Then the function $h:(\mathrm{ri} A) \times Y_{0} \rightarrow \mathbf{R}$ defined by $h(p, q)=\rho_{A-p}(q)$ for $(p, q) \in($ ri $A) \times$ $Y_{0}$ is locally Lipschitz.

Proof. Without loss of generality we may assume that $Y_{0}=Y$ and then ri $A=$ int $A$. Take $p_{0} \in$ int $A, q_{0} \in Y$ and let $r>0$ be such that $p_{0}+r B \subseteq A$. Put $U \equiv p_{0}+2^{-1} r B$ and take $p \in U$. For every $q_{1}, q_{2} \in Y$ we have that

$$
\begin{aligned}
\left|\rho_{A-p}\left(q_{1}\right)-\rho_{A-p}\left(q_{2}\right)\right| & \leqslant \max \left\{\rho_{A-p}\left(q_{1}-q_{2}\right), \rho_{A-p}\left(q_{2}-q_{1}\right)\right\} \\
& \leqslant \rho_{2^{-1} r B}\left(q_{1}-q_{2}\right)=2 r^{-1}\left\|q_{1}-q_{2}\right\| .
\end{aligned}
$$

In particular

$$
\left|\rho_{A-p}(q)\right| \leqslant 2 r^{-1}\|q\| \quad \text { for any } q \in Y .
$$

Let $V \equiv q_{0}+s B$ be a fixed neighbourhood of $q_{0}$, for some $s>0$, and let $q \in V$. Take $p_{1}, p_{2} \in U$. If $q \in t\left(A-p_{2}\right)$ for some $t>0$ then

$$
\begin{aligned}
q & \in t\left(A-p_{1}\right)+t\left(p_{1}-p_{2}\right) \\
& \subseteq t\left(A-p_{1}\right)+t\left(\rho_{A-p_{1}}\left(p_{1}-p_{2}\right)+\varepsilon\right)\left(A-p_{1}\right) \\
& =t\left(1+\rho_{A-p_{1}}\left(p_{1}-p_{2}\right)+\varepsilon\right)\left(A-p_{1}\right), \quad \text { for every } \varepsilon>0 .
\end{aligned}
$$

Hence, for every $\varepsilon>0$, we obtain that

$$
\left(1+\rho_{A-p_{1}}\left(p_{1}-p_{2}\right)+\varepsilon\right)^{-1} q \in t\left(A-p_{1}\right),
$$

which proves that

$$
\rho_{A-p_{1}}\left(\left(1+\rho_{A-p_{1}}\left(p_{1}-p_{2}\right)+\varepsilon\right)^{-1} q\right) \leqslant \rho_{A-p_{2}}(q) .
$$


Thus, we must actually have that

and

$$
\rho_{A-p_{1}}(q) \leqslant\left(1+\rho_{A-p_{1}}\left(p_{1}-p_{2}\right)\right) \rho_{A-p_{2}}(q)
$$

$$
\rho_{A-p_{1}}(q)-\rho_{A-p_{2}}(q) \leqslant \rho_{A-p_{1}}\left(p_{1}-p_{2}\right) \rho_{A-p_{2}}(q) .
$$

Replacing $p_{1}$ with $p_{2}$ we end up with the following inequality

$$
\begin{aligned}
\mid \rho_{A-p_{2}} & (q)-\rho_{A-p_{1}}(q) \mid \\
& \leqslant \max \left\{\rho_{A-p_{2}}(q) \rho_{A-p_{1}}\left(p_{1}-p_{2}\right), \rho_{A-p_{1}}(q) \rho_{A-p_{2}}\left(p_{2}-p_{1}\right)\right\} \\
& \leqslant 2 r^{-1}\|q\| 2 r^{-1}\left\|p_{1}-p_{2}\right\| \\
& \leqslant 4 r^{-2}\left(s+\left\|q_{0}\right\|\right)\left\|p_{1}-p_{2}\right\|,
\end{aligned}
$$

using $\left(1^{\prime}\right)$ and the fact that $p_{1}, p_{2} \in U$.

Finally take $\left(p_{i}, q_{i}\right) \in U \times V, i=1,2$. Then, using (1) and (2) we obtain that

$$
\begin{aligned}
\left|h\left(p_{1}, q_{1}\right)-h\left(p_{2}, q_{2}\right)\right| & \leqslant\left|\rho_{A-p_{1}}\left(q_{1}\right)-\rho_{A-p_{1}}\left(q_{2}\right)\right|+\left|\rho_{A-p_{1}}\left(q_{2}\right)-\rho_{A-p_{2}}\left(q_{2}\right)\right| \\
& \leqslant L\left(\left\|p_{1}-p_{2}\right\|+\left\|q_{1}-q_{2}\right\|\right),
\end{aligned}
$$

where $L=\max \left\{2 r^{-1}, 4 r^{-2}\left(s+\left\|q_{0}\right\|\right)\right\}$, which proves that $h$ is Lipschitz continuous on $U \times V$.

Let us note that the function $h$ in the above lemma may be convex in no neighbourhood of $\left(p_{0}, q_{0}\right) \in$ (int $\left.A\right) \times Y$. An example can be provided with the same subset $f(X)-C=]-\infty, 1]$ in $\mathbf{R}$ and $p_{0}=0, q_{0}=1$ as that was used for the function $m$.

Proposition 3.1. Let $A \equiv f(X)-C$ be convex and closed with ri $A \neq \varnothing$. Let $\left(p_{0}, q_{0}\right) \in M$ be such that $\left(p_{0}+\mathbf{R} q_{0}\right) \cap \operatorname{ri} A \neq \varnothing$. Then the marginal function $m$ is locally Lipschitz at $\left(p_{0}, q_{0}\right)$ on $\left(p_{0}+Y_{0}\right) \times \operatorname{span} C$. If, moreover, $\operatorname{span} C=Y$ then $m$ is locally Lipschitz at $\left(p_{0}, q_{0}\right)$ on $Y \times Y$.

Proof. Let $p_{0}+t_{0} q_{0} \in \mathrm{ri} A$ for some $t_{0} \in \mathbf{R}$. It is easy to check that for every $(p, q) \in\left(p_{0}+Y_{0}\right) \times \operatorname{span} C$ we have $m\left(p-t_{0} q, q\right)=m(p, q)+t_{0}$, with the usual convention that $\pm \infty+t_{0}= \pm \infty$. Hence $\left(p_{0}+t_{0} q_{0}, q_{0}\right) \in M$ if and only if $\left(p_{0}, q_{0}\right) \in M$ and Lipschitz continuity of $m$ at $\left(p_{0}+t_{0} q_{0}, q_{0}\right)$ on $\left(p_{0}+Y_{0}\right) \times$ span $C$ together with span $C \subseteq Y_{0}$ implies that $m$ is locally Lipschitz at $\left(p_{0}, q_{0}\right)$ on $\left(p_{0}+Y_{0}\right) \times \operatorname{span} C$ as well. Thus without loss of generality we may assume that $p_{0} \in$ ri $A$. Then by Lemma 3.1 the function $h$ : (ri $\left.A\right) \times \operatorname{span} C \rightarrow \mathbf{R}$ is Lipschitz continuous on some neighbourhood of $\left(p_{0}, q_{0}\right)$ in $\left(p_{0}+Y_{0}\right) \times \operatorname{span} C$. Since $m(p, q)=(h(p, q))^{-1}$ for any $(p, q) \in($ ri $A) \times \operatorname{span} C$ with $h(p, q) \neq 0$ and $h\left(p_{0}, q_{0}\right) \neq 0$ we must actually have that $m$ is Lipschitz continuous on some neighbourhood of $\left(p_{0}, q_{0}\right)$ in $\left(p_{0}+Y_{0}\right) \times \operatorname{span} C$ as well. 
If $\operatorname{span} C=Y$ then $Y_{0}=Y$; this observation completes the proof of Proposition 3.1.

Let $g: M \rightarrow Y$ denote the mapping defined by $g(p, q)=p+m(p, q) q$ for $(p, q) \in M$. Let us note that $g(p, q) \in \partial(f(X)-C)$ for any $(p, q) \in M$, where the symbol $\partial$ denotes the relative topological boundary in $Y$.

The next corollary is straightforward.

COROLlary 3.1. Under the assumptions of Proposition $3.1 \mathrm{~g}$ is locally Lipschitz at $\left(p_{0}, q_{0}\right)$ on $\left(p_{0}+Y_{0}\right) \times \operatorname{span} C$. If moreover $\operatorname{span} C=Y$ then $g$ is locally Lipschitz at $\left(p_{0}, q_{0}\right)$ on $Y \times Y$.

Let us note that we do not assume in Proposition 3.1 and its corollary that int $C \neq \varnothing$.

Following [20] we say that the line segment $[y, z]$ with $y \neq z$ is parallel to $C$ if $z-y \in C \cup(-C)$.

The next proposition is proved in [20].

Proposition 3.2. Let: (*) $f(X)-C$ be convex and closed and let $\left(p_{0}, q_{0}\right) \in M$, and suppose that there exists $Q \subseteq Y$ with $g\left(p_{0}, q_{0}\right) \in$ int $Q$ such that $\partial(f(X)-C)$ $\cap Q$ contains no line segment parallel to $C$. Then any $x \in X$ obtained by solving $P\left(p_{0}, q_{0}\right)$ is a C-maximal point of $f$ on $X$. Moreover, $g\left(p_{0}, q_{0}\right) \in e_{C}(f(X))$, that is, $g\left(p_{0}, q_{0}\right)$ is a $C$-maximal value for $(P)$ and $f(x)=g\left(p_{0}, q_{0}\right)$.

Let us observe that the condition that $\partial(f(X)-C) \cap Q$ contains no line segment parallel to $C$, ensures that we cannot move from $g\left(p_{0}, q_{0}\right)$ in a direction of $-c \neq 0$ with $c \in C$, while still being on the relative boundary of $f(X)-C$.

Combining Corollary 3.1 and Proposition 3.2 we obtain Lipschitz dependence of maximal values on the parameter $(p, q)$.

TheORem 3.1. Let us suppose that the assumptions of Proposition 3.1 and (*) in Proposition 3.2 hold at some $\left(p_{0}, q_{0}\right) \in M$. Then there exist a neighbourhood $U$ of $p_{0}$ in $p_{0}+Y_{0}$ and a neighbourhood $V$ of $q_{0}$ in span $C$ such that $g(p, q) \in e_{C}(f(X))$ for $(p, q) \in U \times V$. Hence there exists a Lipschitz continuous mapping on $U \times V$, whose values are maximal values for $(P)$. If $\operatorname{span} C=Y$ the above $U$ and $V$ can be taken in $Y$.

Proof. By Corollary 3.1 there exists a neighbourhood $U \times V$ of $\left(p_{0}, q_{0}\right)$ in $\left(p_{0}+Y_{0}\right) \times \operatorname{span} C$, such that $g$ is Lipschitz continuous on $U \times V$. We may choose $U \times V$ so that $g(p, q) \in$ int $Q$ for $(p, q) \in U \times V$, where $Q$ is the set given in the assumptions of Proposition 3.2. Applying Proposition 3.2 to each 
$g(p, q)$ with $(p, q) \in U \times V$ we obtain that $g(p, q) \in e_{C}(f(X))$, whenever $(p, q) \in U \times V$, which completes the proof.

\section{Lipschitz dependence of solutions on the parameter $(p, q)$}

In this section we turn our attention to solutions obtained by solving $P(p, q)$ for $(p, q)$ sufficiently close to given $\left(p_{0}, q_{0}\right)$. Precisely, we want to prove the existence of a Lipschitz continuous mapping defined on some neighbourhood of $\left(p_{0}, q_{0}\right)$ in $M$ whose values are optimal solutions to $(\mathrm{P})$. To this end we shall assume that the mapping $f$ admits locally a Lipschitz continuous inverse.

Let us recall that a multifunction $F: Z_{1} \rightarrow Z_{2}$, where $Z_{1}$ and $Z_{2}$ are topological spaces, is said to be upper continuous (u.c.) at some $z_{1} \in Z_{1}$ if for every open set $Q \subseteq Z_{2}$ containing $F\left(z_{1}\right)$ there exists a neighbourhood $V$ of $z_{1}$ such that $F(z) \subseteq Q$ for every $z \in V$.

The reader can find studies on topological properties of multifunctions in $[2,5,9]$, for instance. However, usually the terminology varies from author to author.

Following [20] we denote by $S: Y \times \operatorname{span} C \rightarrow Y$ the solution multifunction, that is, for $(p, q) \in Y \times \operatorname{span} C$

$$
S(p, q)=\{x \in X: f(x)=p+m(p, q) q+c, \text { for some } c \in C\} .
$$

Conditions ensuring that $S$ is u.c. at $\left(p_{0}, q_{0}\right)$ are given in [20, Corollary 6.2]. For instance, we may observe here that $S$ is u.c. at $\left(p_{0}, q_{0}\right) \in M$ whenever the marginal function $m$ is continuous at $\left(p_{0}, q_{0}\right), X$ is compact and $f$ is continuous.

From now on we shall assume that $X$ is a subset of a topological space $Z$ and $f$ is defined on some neighbourhood $G$ of $X$ in $Z$.

THEOREM 4.1. Let the assumptions of Theorem 3.1 hold at some $\left(p_{0}, q_{0}\right) \in M$ with the multifunction $S$ being u.c. at $\left(p_{0}, q_{0}\right)$. Moreover, suppose that $Z$ is a normed space, $f$ is a homeomorphism on some neighbourhood of $S\left(p_{0}, q_{0}\right)$ in $Z$ and its inverse is locally Lipschitz. Then there exist neighbourhoods $U$ of $p_{0}$ in $p_{0}+Y_{0}$ and $V$ of $q_{0}$ in span $C$ and Lipschitz continuous mapping $s: U \times V \rightarrow X$ such that $s(p, q)$ is a $C$-maximal point of $f$ on $X$ for each $(p, q) \in U \times V$. If $\operatorname{span} C=Y$ then $U$ and $V$ can be taken in $Y$.

Proof. Under the assumptions of Theorem 3.1 and using the fact that $f$ is a homeomorphism on a neighbourhood of $S\left(p_{0}, q_{0}\right)$ we must actually have that $S\left(p_{0}, q_{0}\right)$ consists of only one point. Let $G_{0}$ be a neighbourhood of $S\left(p_{0}, q_{0}\right)$ in $Z$ such that $f: G_{0} \rightarrow f\left(G_{0}\right)$ is a homeomorphism and its inverse $f^{-1}: f\left(G_{0}\right) \rightarrow G_{0}$ 
is Lipschitz continuous on $f\left(G_{0}\right)$. Applying Theorem 3.1 and using the fact that $S$ is u.c. at $\left(p_{0}, q_{0}\right)$ we can find a neighbourhood $U \times V$ of $\left(p_{0}, q_{0}\right)$ in $\left(p_{0}+Y_{0}\right) \times$ span $C$ such that $g$ is Lipschitz continuous on $U \times V, g(p, q) \in e_{C}(f(X))$ and $S(p, q) \subseteq G_{0}$ for $(p, q) \in U \times V$. Under our assumptions it actually follows that if $x \in S(p, q)$ with $(p, q) \in U \times V$ then $g(p, q)=f(x) \in f\left(G_{0}\right)$. Hence the mapping $s: U \times V \rightarrow X$ defined by $s(p, q)=f^{-1} g(p, q)$ for $(p, q) \in U \times V$ is Lipschitz continuous on $U \times V$ and $s(p, q)$ is $C$-maximal for each $(p, q) \in U \times$ $V$, which completes the proof.

Let us note here that we do not require that $f\left(G_{0}\right)$ is open in the above proof.

There exist a number of theorems ensuring that the mapping $f$ admits locally a Lipschitz inverse, see, for example [6, page 30], [3, page 242], [18], [21, page 69].

\section{Differentiable dependence of solutions on the parameter $(p, q)$}

In the previous sections we have shown that under suitable assumptions the maximal values and solutions depend in Lipschitz way on the parameter $(p, q)$. Hence they both admit differentiable properties to some degree.

We shall assume again that $X$ is a subset of a topological space $Z$ and $f$ is defined on some neighbourhood $G$ of $X$ in $Z$.

Let us remind that Theorerms 3.1 and 4.1 yield mappings $g$ and $s$ giving maximal values and maximal points for $(\mathrm{P})$, respectively. In finite dimensions using the Rademacher's theorem [19, page 30] we have immediately the following proposition.

Proposition 5.1. If $Y$ is finite-dimensional and $\left(p_{0}, q_{0}\right) \in M$ then under the assumptions of Theorem 3.1 there exist neighbourhoods $U$ and $V$ of $p_{0}$ in $p_{0}+Y_{0}$ and $q_{0}$ in span $C$, respectively, such that $g$ is Fréchet differentiable almost everywhere on $U \times V$; if also $Z$ is finite dimensional and the assumptions of Theorem 4.1 hold then the same is true for $s$. If, moreover, $\operatorname{span} C=Y$ then the mappings $g$ and $s$ are differentiable almost everywhere in some neighbourhood of $\left(p_{0}, q_{0}\right)$ in $Y \times Y$.

In the above proposition "almost everywhere" is meant in the sense of Lebesgue measure.

There exist a number of generalizations of Rademacher's theorem to infinite dimensions, they can be applied in order to obtain differentiability of $g$ and $s$ when $Y$ and $Z$ are not finite-dimensional. In the rest of this section we shall discuss some of these possibilities. We need the following notation [21].

Let $Z_{1}, Z_{2}$ be topological vector spaces over the reals and let $h: U \rightarrow Z_{2}$ be a mapping defined on some neighbourhood $U$ of $z$ in $Z_{1}$. The mapping $h$ is said to 
be directionally differentiable at $z$ if for every $d \in Z_{1}$

$$
\lim _{t \rightarrow 0} t^{-1}(h(z+t d)-h(z))=h^{\prime}(z ; d)
$$

exists.

If the above $h^{\prime}(z ; \cdot): Z_{1} \rightarrow Z_{2}$ is a continuous linear mapping then we say that $h$ is Gâteaux differentiable at $z$ and we denote $h^{\prime}(z ; \cdot)$ simply by $h^{\prime}(z)$, that is, $h^{\prime}(z)(d)=h^{\prime}(z ; d)$ for every $d \in Z_{1}$. If moreover the convergence in (3) is uniform with respect to $d$ on each compact set in $Z_{1}$ we say that $h$ is Hadamard differentiable at $z$.

It is easily seen that if $Z_{1}$ and $Z_{2}$ are normed spaces and $h$ is a locally Lipschitz mapping at $z$, then it is sufficient for $h^{\prime}(z, \cdot)$ to be linear in order to be Gâteaux differentiable at $z$. Moreover, if a locally Lipschitz mapping at $z$ is Gâteaux differentiable at $z$ then it is Hadamard differentiable at $z$ as well [21, page 19].

THeOREM 5.1. Suppose that $Y$ is a separable Banach space and that $\operatorname{span} C=Y$. Assume that the assumptions of Theorem 3.1 hold at some $\left(p_{0}, q_{0}\right) \in M$. Then $g$ is Hadamard differentiable on a dense subset of some neighbourhood of $\left(p_{0}, q_{0}\right)$ in $Y \times Y$.

Proof. By Proposition 3.1 we obtain that the marginal function $m$ is Lipschitz on a neighbourhood $V$ of $\left(p_{0}, q_{0}\right)$ in $Y \times Y$. As $Y$ is a separable Banach space, the result of Mankiewicz [12, Theorem 4.5] ensures that $m$ is Gâteaux differentiable, hence Hadamard differentiable, on a dense subset of $V$. The Hadamard differentiability of $m$ implies the Hadamard differentiability of the mapping $g$. Applying Theorem 3.1 we get the theorem.

The proof of the next result is analogous, using Theorem 4.1 and Theorem 4.5' in [12].

Theorem 5.2. Assume that $Y$ is a separable Banach space and that $\operatorname{span} C=Y$. Suppose that the assumptions of Theorem 4.1 hold at some $\left(p_{0}, q_{0}\right) \in M$ with $Z$ being a reflexive Banach space. Then $s$ is Hadamard differentiable on a dense subset of some neighbourhood of $\left(p_{0}, q_{0}\right)$ in $Y \times Y$.

Hypotheses on $Z$ other than reflexivity under which Theorem 5.2 still holds can be found in [12, Definition 2.4, Theorem 4.5].

However, Theorems 5.1 and 5.2 extend Proposition 5.1 to infinite dimensions, but their strength is far less, as they ensure differentiability only on a dense subset compared; with "almost everywhere" in Proposition 5.1. Mankiewicz [13, Theorem 3.6] has proved that if $h$ is a Lipschitz mapping from an open subset of a 
separable Banach space $Z_{1}$ to a so-called $G F$-space $Z_{2}$ (for example every reflexive Banach space is $G F$-space) then the set of those points, where $h$ is not Gâteaux differentiable is of "measure zero" in $Z_{1}$. The class of subset of "measure zero" [13] in a separable Banach space used in the above result is defined in such a way that they behave like sets of Lebesgue measure zero in Euclidean space. Aronszajn [1, Theorem 1, page 166] has strengthened the result of Mankiewicz to a class of exceptional subsets, which is more restricted then the class of subsets of "measure zero" in [13]. Applying the above results of Mankiewicz and Aronszajn one may considerably strengthen Theorems 5.1 and 5.2. However, rather than discuss these more complicated situations here, we refer the reader to the above-mentioned papers of Mankiewicz and Aronszajn. See [11] also.

Let us observe that in the above results we have not supposed that $f$ satisfies any differentiability assumptions.

\section{Conclusions}

We end our discussion with the observation that for a quite large class of vector optimization problems set in normed spaces the results of the previous sections can be applied locally. Let us recall the definition of local optimal solutions for the problem (P).

Let $X$ be a topological space and let $f: X \rightarrow Y$ be a continuous mapping. We say that $x \in X$ is a locally $C$-maximal point of $f$ on $X$ if there exists a neighbourhood $N$ of $x$ such that $x$ is a $C$-maximal point of $f$ on $N$.

We can apply the results of the previous sections whenever there exists a neighbourhood $Q$ in $Y$ such that $f(X) \cap Q \neq \varnothing$ and $f(X) \cap Q-C$ is convex and closed. Considering the problem (P) with $X$ replaced by $N=f^{-1}(Q)$ which is a neighbourhood in $X$ in view of the continuity of $f$, we obtain a Lipschitz and differentiable dependence of locally $C$-maximal values and locally $C$-maximal points of $f$ on $X$ on the parameter $(p, q)$. Moreover, observe that we do not assume here that $X$ is convex, in fact the space $Z$, of which $X$ is a subset, need not be a linear space. The mapping $f$ is an arbitrary continuous mapping on $X$. However, let us note that if $C$ is a pointed convex cone (that is, $C \cap(-C)=\{0\}$ ), $f$ is $(-C)$-convex on a convex subset $X$ (that is, $(1-t) f\left(x_{1}\right)+t f\left(x_{2}\right)-$ $f\left((1-t) x_{1}+t x_{2}\right) \in-C$ for every $x_{1}, x_{2} \in X$ and $\left.t \in[0,1]\right)$ then any locally $C$ maximal point of $f$ on $X$ is a globally $C$-maximal point of $f$ on $X$. 


\section{Acknowledgment}

The author would like to thank Professor John Miller for his careful readings of the manuscript and the referee, who suggested many improvements.

\section{References}

[1] N. Aronszajn, 'Differentiability of Lipschitzian mappings between Banach spaces', Studia Math. 57 (1976), 147-190.

[2] C. Berge, Topological spaces (Macmillan, New York, 1963).

[3] F. Clarke, Optimization and nonsmooth analysis (Wiley-Interscience, New York, 1983).

[4] B. D. Craven, 'Vector-valued optimization', Generalized concavity in optimization and economics eds. S. Schaible and W. T. Ziemba, pp. 661-687 (Academic Press, London, 1981).

[5] J. P. Dalahaye and J. Denel, 'The continuities of the point-to-set maps, definitions and equivalences', Math. Programming Study 10 (1979), 8-13.

[6] J. Dugundji and A. Granas, Fixed point theory, Vol. I (PWN-Polish Scientific Publishers, Warszawa, 1982).

[7] M. I. Henig, 'Existence and characterization of efficient decisions with respect to cones', Math. Programming 23 (1982), 111-116.

[8] R. B. Holmes, Geometric functional analysis and its applications, (Springer, New York, New York, 1975).

[9] K. Kuratowski, Topology (Academic Press, New York and Polish Scientific Publishers, Warszawa, 1966).

[10] J. L. Lin, 'Maximal vectors and multiobjective optimization', J. Optim. Theory Appl. 18 (1976), 41-64.

[11] G. Lebourg, 'Generic differentiability of Lipschitzian functions', Trans. Amer. Math. Soc. 256 (1979), 12-144.

[12] P. Mankiewicz, 'On the differentiability of Lipschitz mappings in Fréchet spaces', Studia Math. 45 (1973), 15-29.

[13] P. Mankiewicz, 'On topological, Lipschitz, and uniform classification of $L F$-spaces', Studia Math. 52 (1975), 109-142.

[14] A. Nijenhuis, 'Strong derivatives and inverse mappings', Amer. Math. Monthly 81 (1974), 969-980.

[15] A. Pascoletti and P. Serafini, 'Scalarizing vector optimization problems', J. Optim. Theory Appl., 42 (1984), 499-524.

[16] J. P. Penot and A. Sterna-Karwat, 'Parametrized multicriteria optimization; continuity and closedness of optimal multifunctions', J. Math. Anal. Appl., to appear.

[17] J. P. Penot and A. Sterna-Karwat, 'Parametrized multicriteria optimization; order continuity of the marginal multifunctions', J. Math. Anal. Appl., to appear.

[18] B. H. Pourciau, 'Analysis and optimization of Lipschitz continuous mappings', J. Optim. Theory Appl. 22 (1977), 311-351.

[19] L. Simon, Lectures on geometric measure theory (Centre of Mathematical Analysis, Australian National University, 1984). 
[20] A. Sterna-Karwat, 'Continuous dependence of solutions on a parameter in a scalarization method', J. Optim. Theory Appl., to appear.

[21] S. Yamamuro, Differential calculus in topological linear spaces, (Springer-Verlag, Berlin, 1974).

[22] P. L. Yu, 'Cone convexity, cone extreme points and nondominated solutions in decision problems with multiobjectives', J. Optim. Theory Appl. 14 (1974), 319-317.

\section{Department of Mathematics \\ Monash University \\ Clayton, Victoria 3168 \\ Australia}

